BULLETIN (New Series) OF THE

AMERICAN MATHEMATICAL SOCIETY

Volume 45, Number 3, July 2008, Pages 409-427

S 0273-0979(08)01211-1

Article electronically published on April 9, 2008

\title{
AN EXAMPLE OF ARNOLD DIFFUSION FOR NEAR-INTEGRABLE HAMILTONIANS
}

\author{
VADIM KALOSHIN AND MARK LEVI
}

\begin{abstract}
In this paper, using the ideas of Bessi and Mather, we present a simple mechanical system exhibiting Arnold diffusion. This system of a particle in a small periodic potential can be also interpreted as ray propagation in a periodic optical medium with a near-constant index of refraction. Arnold diffusion in this context manifests itself as an arbitrary finite change of direction for nearly constant index of refraction.
\end{abstract}

\section{INTRODUCTION}

Understanding stability of Hamiltonian systems is one of the oldest and most funamental problems of classical mechanics. For near-integrable Hamiltonian systems, i.e. for Hamiltonian systems "close" to

$$
\dot{I}=0, \dot{\theta}=\omega(I), \text { where } I, \theta \in \mathbb{R}^{n},
$$

a partial solution to the problem is provided by Kolmogorov-Arnold-Moser (KAM) theory: Under certain natural nondegeneracy and smoothness conditions, there exists a positive measure Cantor of invariant $n$-tori in the phase space $\mathbb{R}^{2 n}$. This set approaches full measure as the size of the perturbation becomes small. The KAM tori are close to the unperturbed tori given by $I=$ const, $\theta \in \mathbb{R}^{n}(\bmod 1)$. For any solution starting on an invariant torus, the Hamiltonian action $I(t)$ stays close to $I(0)$ for all time, and one has stability for all time with "high probability", since the invariant tori cover most of the phase volume. In fact, for $n \leq 2$, one has stability for all solutions - not only the ones lying on the tori- since 2-tori confine trajectories on a 3-dimensional energy surface. This topological reason no longer applies for $n \geq 3$, and Arnold conjectured over 40 years ago that a generic near-integrable system has solutions that "diffuse" through the complement of the set of KAM tori in such a way that the action changes by $O(1)$ over a sufficiently long time. More precisely, it was conjectured that there exists an arbitrarily small Hamiltonian perturbation of (1) for which there exist solution(s) $(I(t), \theta(t))$ satisfying

$$
\left|I\left(t_{2}\right)-I\left(t_{1}\right)\right|>A
$$

for some $t_{1}<t_{2}$, where $A>0$ is a constant independent of the size of the perturbation. Such drift of action is now commonly referred to as Arnold diffusion.

Received by the editors March 3, 2007, and, in revised form, September 17, 2007.

2000 Mathematics Subject Classification. Primary $70 \mathrm{H} 08$.

The first author was partially supported by the Sloan Foundation and NSF grants, DMS0701271.

The second author was partially supported by NSF grant DMS-0605878. 
In this note we give a very simple example of Arnold diffusion where the main difficulties of the subject do not appear. This example is a variation on Arnold's example and we treat it by a variational method similar to those of Bessi [6] and Mather [25. A closely related example is described in [17]. We sacrifice the generality and technical strength for the sake of transparency. Our primary goal is to present the geometrical essence of Arnold diffusion in what seems to be the simplest possible setting. A rich literature (see, e.g., 3], 8, [19]) on Arnold diffusion goes far beyond the example discussed here. We outline our construction in this Introduction, giving details in later sections.

Example. Consider a small perturbation of a free particle $\ddot{\theta}=0$ :

$$
\ddot{\theta}=-\varepsilon \nabla U(\theta, \varepsilon), \varepsilon \in \mathbb{R}^{3},
$$

where $U$ is a periodic potential of the form

$$
U(\theta, \varepsilon)=\cos ^{2}\left(\frac{\pi z}{2}\right)-\varepsilon^{k} \beta(\theta, \varepsilon) \text { with } \theta=(x, y, z) \in \mathbb{R}^{3},
$$

with $k \geq 2$ and where $\beta$ is a $C^{\infty}$ smooth "bump" function, periodic in $x, y, z$, supported on $\varepsilon$-balls centered at integer points in $\mathbb{Z}^{3}$ with even coordinates 1

$$
\beta(\theta, \varepsilon)=\sum_{n \in \mathbb{Z}^{3}} \eta\left(\frac{|\theta-2 n|}{\varepsilon}\right),
$$

where, to be specific, we pick $\eta\left(\left[0, \frac{1}{2}\right]\right)=1, \eta([1, \infty))=0$ with $\eta$ being $C^{\infty}$ smooth monotone decreasing on $\left[\frac{1}{2}, 1\right]$, even, and smooth on $\mathbb{R}$. Actually, for our results to hold, $\eta$ can be any smooth nonnegative function whose support is $(-1,1)$. Under these conditions the contribution of the bump term $\varepsilon^{k+1} \beta$ in (2) is indeed $C^{k}$-small with $\varepsilon$. For $\varepsilon=0$, the Hamiltonian system (2) is integrable and there is no diffusion: the action $\dot{\theta}=$ const. We will show that for $\varepsilon>0$, no matter how small, the diffusion is present-more precisely, that there exist trajectories whose velocity (that is, action) changes by $O(1)$ over a sufficiently large time. This velocity stays, as it turns out, nearly parallel to the $(x, y)$-plane and changes direction there. This change in a particular direction is a manifestation of the general phenomenon: the action drifts along resonances. The drift is due to the failure of averaging: resonant trajectories are not well distributed in phase space. In our case the resonant frequency $\omega=\left(\omega_{1}, \omega_{2}, 0\right)$ corresponds to the motion in the horizontal plane and satisfies the resonance relation $k \cdot \omega=0$ with an integer vector $k=\left(0,0, k_{3}\right)$.

A brief survey of results on Arnold diffusion. In his famous paper [2], Arnold gave an example of a near-integrable system exhibiting diffusion. Arnold conjectured (see, e.g., 3]) that a generic perturbation of a completely integrable system 2 exhibits diffusion. Later Bessi [6] analyzed the speed of diffusion in Arnold's example using a variational method; see also Berti and Bolle 8 for an extensive list of further references.

Trajectories of a completely integrable system (2) with $\varepsilon=0$ come in three different types: quasiperiodic (whose closures are $\mathbb{R}^{3}(\bmod 1)$ ), resonant quasiperiodic (whose closures are 2-dimensional tori), and periodic (1-dimensional tori). In the first case, $\dot{\theta}=\omega$ is an irrational vector; in the second case $\omega \cdot k=0$ for some

\footnotetext{
${ }^{1}$ This choice is made for later convenience.

${ }^{2}$ In this case it is of at least 2.5 degrees of freedom.
} 


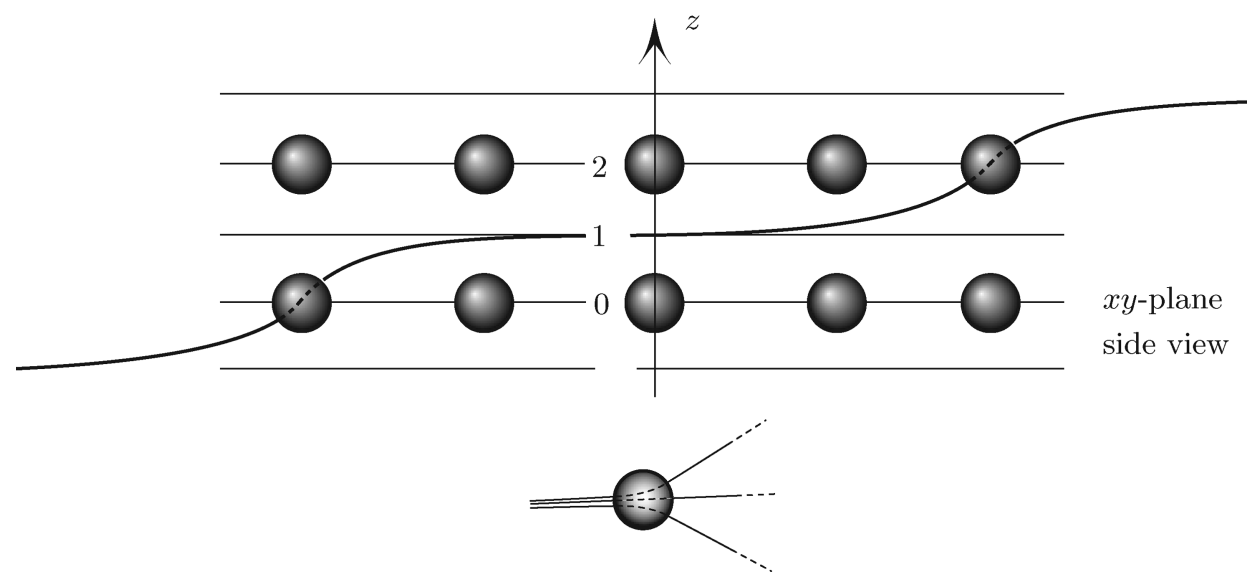

FiguRE 1. A: Side view; B: Scattering property of the "scatterer";

C: Drift of action.

$k \in \mathbb{Z}^{3} \backslash\{0\}$; and in the last case $\omega \cdot k_{i} \neq 0, i=1,2$, where $k_{1}$ and $k_{2}$ are noncollinear integer vectors. We refer to the first case as the single resonance and to the second case as the double resonance.

Behavior near the single resonance after perturbation. Fix a nonzero integer vector $k$, and consider the manifold $M_{k}=\{(\theta, I): \omega(I) \cdot k=0\}$ restricted to a 5 -dimensional energy surface. $M_{k}$ is foliated by resonant tori of different types. Under a typical perturbation, most of these tori are destroyed and finitely many normally hyperbolic invariant manifolds of codimension two (within the energy surface) arise in their place. With an appropriate choice of local coordinates near $M_{k}$, the system to the leading order is given by the Hamiltonian

$$
H(J, \phi, p, q, t, \varepsilon)=\underbrace{\frac{J^{2}}{2}}_{\text {rotator }}+\underbrace{\frac{p^{2}}{2}+(\cos q-1)}_{\text {harmonic oscillator }}+\varepsilon H_{1}(J, \phi, p, q, t) .
$$

The rotator describes the dynamics within the normally hyperbolic manifold, while the harmonic oscillator governs the transversal directions. Usually, such a system is referred to as a priori unstable. Arnold's example fits in this class. This a priori unstable structure is the key property used in a wide range of research on diffusion for generic small time-periodic perturbations of (5). Recently, great progress has been achieved in proving diffusion in this case. Bernard 4, Cheng and Yan 10, and Xia 31 perform thorough studies of this example using different powerful variational approaches influenced by the work of Mather [21. Delshams, de la Llave and Seara 13 apply the classical Arnold method based on transition chains. The beautiful new idea there is to use not only classical "primary" tori, but also "secondary" tori. In [29] Treschev investigates "separatrix maps", i.e., maps along a homolinic orbit, and in [30, based on properties of these maps, he not only proves existence of diffusing orbits but also shows that the optimal diffusion speed is $\varepsilon \log 1 / \varepsilon$.

Arnold's example (50) has also been generalized to the case of higher dimension $(p, q) \in \mathbb{R}^{2 k}, k \geq 2$. Cheng and Yan [11] studied this case using the same variational method as in [10] and proved the existence of diffusion. 
Double resonance. Doubly resonant tori corresponding to a fixed rational vector $\omega$ is foliated by a two-parameter family of periodic orbits in the unperturbed system. A typical perturbation destroys all but finitely many of these periodic orbits, some of which become hyperbolic, with 3-dimensional stable and 3-dimensional unstable manifolds. Analysis of diffusion in the neighborhood of doubly resonant tori is substantially more difficult, since there is no obvious direction of diffusion as in the case of single resonance, where diffusion took place along the cylinder $C$. This more difficult case is referred to as "a priori stable". Mather in [22] announced proof of existence of Arnold diffusion for time-periodic perturbations of convex a priori stable integrable systems of two degrees of freedom. Proof of this fundamentally important result is highly involved and a substantial part of it is available in 25 and [26]. A method of proof is called by Mather a method of changing Lagrangians 3 One of the goals of this paper is to illustrate this method in the simplest possible situation. Based on the proof presented below, we then sketch the general scheme of Mather's method.

Showing Arnold diffusion for a particular family of examples is an important step toward proving Arnold's conjecture. Marco and Sauzin [19, in a collaboration started with Herman, presented such a family in the class of Gevrey near-integrable Hamiltonians.

Apart from the delicate question of existence of diffusion, it is important to have upper bounds on diffusion speed. The famous Nekhoroshev stability estimates 27. show that for analytic near-integrable Hamiltonian systems with quasiconvex unperturbed Hamiltonian, diffusion time is at least exponentially long. Marco and Sausin [19] extend Nekhoroshev estimates to the Gevrey class and construct a family of examples in this class with optimal, i.e., maximal possible, speed of diffusion. In 9] Bourgain and Kaloshin give an open family of analytic examples which possess trajectories diffusing with optimal speed, thus proving optimality of the Nekhoroshev stability estimates [27. In the present paper we give a family of examples of a smooth near-integrable Hamiltonian system having polynomial speed of diffusion 5

A heuristic explanation of diffusion. Before presenting rigorous details, we give a heuristic description of diffusion in our example, illustrating along the way the more general concepts of Mather's general approach to studying diffusion. Consider first the potential (3) without the $\beta$-term. The "odd" planes $z=2 m+1, m \in \mathbb{Z}$, "repel" most of the trajectories of (2), since the potential is miminal on these planes. Now the $\beta$-term in the potential (3) "pulls" action minimizers toward the center of the scatterer. This "pull" is instrumental in constructing diffusion. Figure 1 illustrates how these consecutive deflections can accumulate.

\footnotetext{
${ }^{3}$ See 9 for an application of this method to twist maps and to the problem of existence of diffusion for an open set of a priori stable nearly integrable systems.

${ }^{4}$ The class of Gevrey functions contains $C^{\infty}$ functions and is contained in the class of functions analytic in an arbitrary complex strip.

${ }^{5}$ Our example could be extended not only to an arbitrary number of degrees of freedom, but also to analytic perturbations. This, however, would lead to technical complications, which we want to avoid.
} 
The MAIN RESUlT AND THE VARIATIONAL FORMULATION

Theorem 1. There exists $\varepsilon_{0}>0$ such that for all $0<\varepsilon \leq \varepsilon_{0}$ the flow of (2) has the following properties.

- For any two limiting velocities $c=\left(c_{1}, c_{2}, 0\right),|c|=1$, and $c^{\prime}=\left(c_{1}^{\prime}, c_{2}^{\prime}, 0\right)$, $\left|c^{\prime}\right|=1$, there exists a trajectory $\gamma(t)$ with

$$
\dot{\gamma}(t) \rightarrow c \text { as } t \rightarrow+\infty \text { and } \dot{\gamma}(t) \rightarrow c^{\prime} \text { as } t \rightarrow-\infty \text {; }
$$

- For any $\delta>0$ and for any direction $c=\left(c_{1}, c_{2}, 0\right),|c|=1$, there is a "direction-reversing" trajectory $\gamma(t)$ of (2) such that for some $t_{-}<t_{+}$we have $\left|\gamma\left(t_{-}\right)-\gamma\left(t_{+}\right)\right|<\delta$ and $\left|\dot{\gamma}\left(t_{-}\right)+\dot{\gamma}\left(t_{+}\right)\right|<\delta$.

Remark 1.1. The restriction on the limiting speeds to equal 1 can be removed by rescaling time.

Existence of direction changing trajectories is proven by constructing shadowing orbits in Lemma 4 .

Our system (2) is the Euler-Lagrange equation for the Lagrangian

$$
L(\theta, \dot{\theta}, \varepsilon)=\frac{\langle\dot{\theta}, \dot{\theta}\rangle}{2}+\varepsilon U(\theta ; \varepsilon), \theta=(x, y, z) \in \mathbb{R}^{3} .
$$

We introduce the "scatterers" as the balls whose union contains the support of $\beta$ :

$$
\mathcal{L}_{n}=\{\theta:|\theta-2 n|<\sqrt{\varepsilon}\}, n \in \mathbb{Z}^{3} .
$$

Since $(-\beta)<0$ inside the scatterers, we expect the minimizers of the action $\int L d t$ passing through the scatterers to be "attracted" to their center.

The proof of the main theorem, Theorem 1, consists of two parts. First, following ideas of Mather and Peierls, we define the Mather-Peierls' barrier function. Second, we use the barrier function to define a la Mather a variational functional (see (29) ) which we then show to have a minimizer 6 This minimizer has the desired "shadowing" properties as will be seen later; however, the solution does not satisfy the Euler-Lagrange equation of $L$. We then show that near this "nonsolution" there exists a solution of the Euler-Lagrange equation of $L$ satisfying the conditions below of the main theorem (see Lemma 4).

\section{Properties OF ASYMPTOTIC TRAJECTORIES}

We point out several basic properties of the flow associated with the Lagrangian $L$.

I. Outside the "scatterers" (7), the flow is completely integrable:

A)

$$
\ddot{x}=\ddot{y}=0, \quad \ddot{z}=\frac{\varepsilon \pi}{2} \sin (\pi z),
$$

and in particular the projection of any trajectory onto the $x y$-plane is a straight segment with uniform motion on it.

B) The projection of any trajectory onto the $z$-axis coincides with a trajectory of the Euler-Lagrange flow of the pendulum,

$$
L^{z}(z, \dot{z})=\frac{\dot{z}^{2}}{2}+\varepsilon \cos ^{2}\left(\frac{\pi z}{2}\right)
$$

\footnotetext{
${ }^{6}$ Ideas in the present paper are also somewhat similar to those in [6].
} 


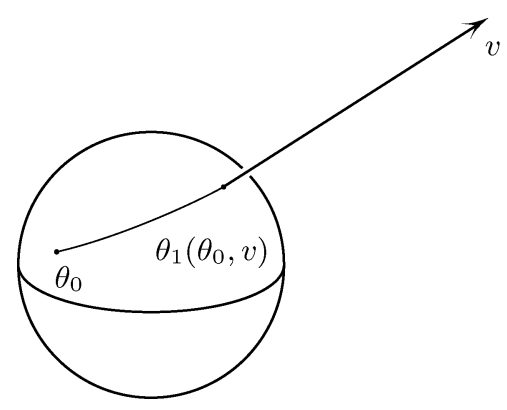

Figure 2. Initial point $\theta_{0}$ and exit velocity $v$ define the "exit" point $\theta_{1}$.

(see Figure 3) with conserved energy

$$
H^{z}(z, \dot{z})=\frac{\dot{z}^{2}}{2}-\varepsilon \cos ^{2}\left(\frac{\pi z}{2}\right) .
$$

II. Inside any scatterer, e.g., $\mathcal{L}_{0}$, we have the following:

A) Let $\theta_{0} \in \operatorname{Int} \mathcal{L}_{0}$ and $v \in \mathbb{R}^{3} \backslash\{0\}$. By the implicit function theorem and the theorem on the dependence of solutions of ODEs, on the inital values there exists a unique trajectory which starts at $\theta_{0}$ and exits $\mathcal{L}_{0}$ with the prescribed velocity $v$, provided $\varepsilon$ is sufficiently small. Furthermore, the exit point $\theta_{1}$ depends smoothly on $\theta_{0}$ and $v$, with $\frac{\partial \theta_{1}}{\partial \theta_{0}}, \frac{\partial \theta_{1}}{\partial v}$ bounded by an $\varepsilon$-independent constant (see Figure 2).

B) For any trajectory in $\mathcal{L}_{0}$, we have

$$
\ddot{x}=O\left(\varepsilon^{k}\right), \ddot{y}=O\left(\varepsilon^{k}\right), \ddot{z}=O(\varepsilon) .
$$

In order to single out solutions with preferred velocities, we define, following Fathi [14, the $c$-Lagrangian,

$$
L_{c}(\theta, \dot{\theta}, \varepsilon)=\frac{\langle\dot{\theta}-c, \dot{\theta}-c\rangle}{2}+\varepsilon U(\theta, \varepsilon)
$$

where $c=$ const, the Euler-Lagrange flows of $L$ and $L_{c}$ are the same, and thus the two variational principles $\int L_{c}$ and $\int L$ have the same critical points. However, $\int L_{c}$, unlike $\int L$, is minimized along trajectories which in some sense follow the direction $c$.

For $\theta_{0}, \theta_{1} \in \mathbb{R}^{3}$, we define

$$
A_{c}^{t}\left(\theta_{0}, \theta_{1}\right)=\inf _{\gamma} \int_{0}^{t} L_{c}(\gamma(s), \dot{\gamma}(s), \varepsilon) d s,
$$

where the infinum is taken over all absolutely continuous curves $\gamma:[0, t] \rightarrow \mathbb{R}^{3}$ with $\gamma(0)=\theta_{0}$ and $\gamma(t)=\theta_{1} 7$ Note that for any $\gamma$ and $t \in \mathbb{R}$ (not necessarily positive) we have

$$
A_{c}^{t}\left(\theta_{0}, \theta_{1}\right)=A_{0}^{t}\left(\theta_{0}, \theta_{1}\right)+\left\langle c, \theta_{1}-\theta_{0}\right\rangle+\frac{\langle c, c\rangle}{2} t .
$$

We also define the one-sided action

$$
A_{c}^{+}\left(\theta_{0}\right)=\liminf _{t \rightarrow \infty} \min _{\theta_{1}} A_{c}^{t}\left(\theta_{0}, \theta_{1}\right) .
$$

\footnotetext{
${ }^{7}$ In what follows we only consider the case when $z$-components of $\theta_{0}$ and $\theta_{1}$ differ by 2 .
} 

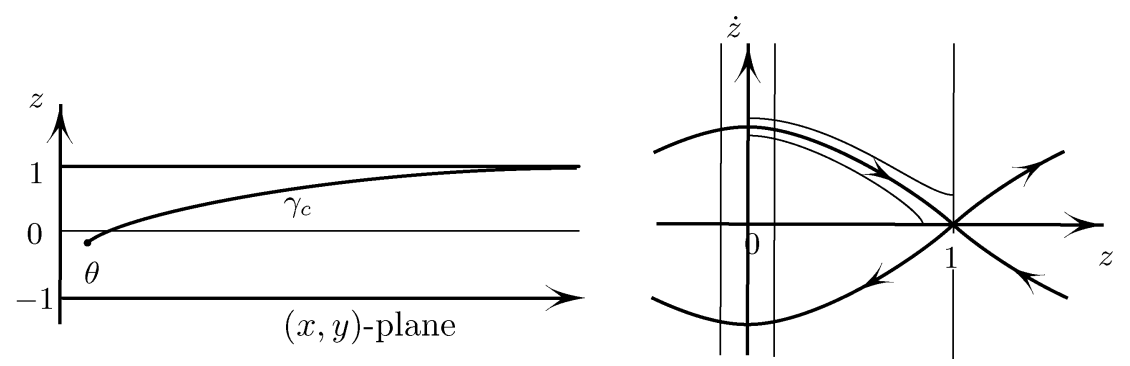

FIgURE 3. Homoclinic trajectory $\gamma_{c}^{+}$in two projections.

From now on, we choose $c=\left(c_{1}, c_{2}, 0\right)$ with $|c|=1$. We will show in Lemma 1 the existence of a trajectory $\gamma_{c}^{+}\left(t, \theta_{0}\right)=(x(t), y(t), z(t))$ with the properties $\gamma_{c}^{+}\left(0, \theta_{0}\right)=\theta_{0}$ and

$$
-1<z(0) \leq 1, \quad \dot{z} \geq 0, \quad z \rightarrow 1, \quad \dot{\gamma}_{c}^{+} \rightarrow c \quad \text { as } t \rightarrow \infty .
$$

We shall prove that $\int_{0}^{\infty} L\left(\gamma_{c}^{+}\left(t, \theta_{0}\right), \dot{\gamma}_{c}^{+}\left(t, \theta_{0}\right)\right) d t$ equals $A_{c}^{+}\left(\theta_{0}\right)$. This will justify calling such a trajectory a one-sided c-minimizer.

Lemma 1. For all small $\varepsilon>0$ the following holds.

(1) Given any $c$ as above, and any $\theta$ in the scatterer $\mathcal{L}_{0}$, there exists a unique one-sided c-minimizer $\gamma_{c}^{+}=\gamma_{c}^{+}(t, \theta)$ with $\gamma_{c}^{+}(0, \theta)=\theta$-more precisely, $\gamma_{c}^{+}$ minimizes (13).

(2) The action $A_{c}^{+}(\theta)$ is 2-periodic and smooth in $\theta$ and smooth in c. Moreover, $A_{c}^{+}$takes a minimum in $\theta$ at planes $z=2 m+1, m \in \mathbb{Z}$. Furthermore, the $C^{1}$-norm of $A_{c}^{+}(\theta)$ in $c$ is bounded uniformly in $\varepsilon$. Similarly, replacing the condition in (14) by $-1 \leq z(0)<1, \dot{z} \geq 0$, and $z \rightarrow-1$ as $t \rightarrow-\infty$ we can define $\gamma_{c}^{-}$and $A_{c}^{-}$.

(3) If $t>\frac{1}{2}\left|\theta_{1}-\theta_{0}\right|>\varepsilon^{-k-3}$, then the minimizer $\theta(t)$ of (12) is $z$-monotone and unique. Moreover, it does not intersect any scatterers except for the ones containing $\theta_{0}, \theta_{1}$. In what follows, we will only consider the case when $\theta_{0}$ and $\theta_{1}$ lie in the neighboring "layers", i.e., $z_{0}=O(\sqrt{\varepsilon})$ and $z_{1}=2+O(\sqrt{\varepsilon})$. Similarly, the one-sided minimizers meet at most one scatterer, namely, the one containing $\theta$.

Remark 1.2 (Whiskered tori and the Peierls barrier).

(1) The union of trajectories $\left(\gamma_{c}^{+}(\theta, t), \dot{\gamma}_{c}^{+}(\theta, t)\right) \in T \mathbb{T}^{3}$ forms a stable manifold $W^{s}\left(\mathbb{T}_{c}^{2}\right)$ of the 2-torus $\mathbb{T}_{c}^{2}=\left\{\dot{x}=c_{1}, \dot{y}=c_{2}, \dot{z}=0, z=1(\bmod 2)\right\} \subset$ $T \mathbb{T}^{3}$. This torus is the counterpart of Arnold's "whiskered torus". The union of $\left(\gamma_{c}^{-}(\theta, t), \dot{\gamma}_{c}^{-}(\theta, t)\right) \in T \mathbb{T}^{3}$ forms an unstable manifold $W^{u}\left(\mathbb{T}_{c}^{2}\right)$. This represents the hidden a priori unstable structure of our example. The variational functional (12) allows us to single out $W^{u}\left(\mathbb{T}_{c}^{2}\right)$.

(2) The function $A_{c}(\theta)$ is an example of the Peierls barrier. It general, this function is Lipschitz $\theta$, but not necessarily smooth or Lipschitz in $c$. In 22$]$ Mather shows that in the simplest nontrivial case of twist maps, $A_{c}(\theta)$ has moduli of continuity with respect to $c$. The regularity of $A_{c}(\theta)$ in $c$ and $\theta$ is fundamental for Mather's variational approach. 
To point out the analogues of "wiskered tori" in our case, or in Mather's method of a changing Lagrangian, consider the so-called projected 8 Aubry set. Ignoring subtleties of the general definition (see, e.g., [23]) which play no role in this example, we let

$$
\hat{\mathcal{A}}_{c}=\left\{\theta^{\prime} \in \mathbb{R}^{3}: A_{c}\left(\theta^{\prime}\right)=\min _{\theta} A_{c}(\theta)\right\} .
$$

Due to periodicity, $\mathcal{A}_{c}=\hat{\mathcal{A}}_{c} / 2 \mathbb{Z}^{3} \subset \mathbb{T}^{3}$ is well defined and is called an Aubry set of cohomology class $c \in R^{3} \simeq H^{1}\left(\mathbb{T}^{3}, \mathbb{R}\right.$ ) (see Section 3 for more). In our example the Aubry set consists of the aforementioned straight lines inside $\mathbb{T}_{c}^{2}$, and its projection $\hat{\mathcal{A}}_{c}$ onto the configuration space is a 2dimensional torus. However, in general $\mathcal{A}_{c}$ may be a Cantor set. The set $\mathcal{A}_{c}$ corresponds to the hyperbolic "whiskered torus".

Proof of Lemma 1. There are two key ideas in the proof below. Consider the truncated Lagrangian without the scattering potential:

$$
L_{0}=\frac{\langle\dot{\theta}, \dot{\theta}\rangle}{2}+\varepsilon U_{0}, U_{0}=\cos ^{2}\left(\frac{\pi z}{2}\right) .
$$

First, notice that the Euler-Lagrange equation of $L_{0}$ is explicitly integrable. This shows existence and uniqueness of the minimizers of $A_{c}^{t}\left(\theta_{0}, \theta_{1}\right)$ for any $t>0$ and any pair $\theta_{0}, \theta_{1}$ whose $z$-components differ at most by 2 . Due to the explicit nature of such minimizers, taking a limit as $t \rightarrow \infty$ is straightforward and gives the existence of one-sided minimizers.

Second, we insert "lenses" by subtracting $\varepsilon^{k} \beta(\theta, \varepsilon)$ from the potential $U$ (see (3)). Due to the smallness of this perturbation, we can apply the implicit function theorem and still prove existence and uniqueness of such minimizers.

We start by proving parts (1) and (2) of Lemma 1. Denote $\theta=(x, y, z)$.

(1) The unperturbed case. Denote by $\gamma_{c}^{0}\left(t, \theta_{0}\right)=\left(X^{0}\left(t, \theta_{0}\right), Y^{0}\left(t, \theta_{0}\right), Z^{0}\left(t, \theta_{0}\right)\right)$, $t \geq 0$ the trajectory with the following properties. It starts at $\theta_{0}$, i.e., $\gamma_{c}^{+}\left(t, \theta_{0}\right)=\theta_{0}$; the $x y$-component of its velocity is constant and equals $\left(c_{1}, c_{2}\right)$, i.e., $\left(X^{0}\left(t, \theta_{0}\right), Y^{0}\left(t, \theta_{0}\right)=(x, y)+\left(c_{1}, c_{2}\right) t\right.$; and the $z$-component belongs to the separatrix in the $(z, \dot{z})$-plane, i.e., $H^{z}\left(Z^{0}\left(t, \theta_{0}\right), \dot{Z}^{0}\left(t, \theta_{0}\right)\right)=$ 0 .

We now show that $\gamma_{c}^{0}\left(t, \theta_{0}\right)$ is indeed a one-sided $c$-minimizer. Notice that semi-infinite action along $\gamma_{c}^{0}\left(\cdot, \theta_{0}\right)$ is finite, therefore, $A_{c}^{+}\left(\theta_{0}\right)$ is finite. By Tonelli's theorem for any $t$ and any $\theta_{1}$, there is $\gamma_{t}:[0, t] \rightarrow \mathbb{R}^{3}$ which minimizes the right-hand side of (11). Then for the $z$-component $z(s)=$ $\pi_{z}\left(\gamma_{t}(s)\right), 0 \leq s \leq t$, we have $A_{c}^{t}\left(\theta_{0}, \theta_{1}\right) \geq \int_{0}^{t} L^{z}(z(s), \dot{z}(s), \varepsilon) d s$. Equality corresponds to the $x y$-component having constant velocity $\left(c_{1}, c_{2}\right)$. The Euler-Lagrange flow of $L^{z}$ is integrable (see Figure 3). It is easy to see that for each $t>0$ there is $\theta_{1}^{*}$ which realizes the minimum $\min _{\theta_{1}} A_{c}^{t}\left(\theta_{0}, \theta_{1}\right)$. Moreover, the $z$-component of a corresponding minimizer has to belong to some energy surface $\left\{H^{z}=E_{t}\right\}$ for some $E_{t}$. Note that $E_{t} \rightarrow 0$ as $t \rightarrow \infty$; otherwise, $L^{z}$ is bounded from below and $A_{c}^{t}$ grows linearly in time. This is a contradiction with finiteness of $A_{c}^{+}\left(\theta_{0}\right)$. To conclude, $\gamma_{c}^{0}$ is the unique one-sided minimizer.

\footnotetext{
${ }^{8}$ onto the configuration space
} 
(2) The perturbed case. Consider the plane $P_{c}$ tangent to $\partial \mathcal{L}_{0}$ and normal to $c$. It suffices to show that for any $\theta_{0} \in \mathcal{L}_{0}$ there exists a unique $\theta_{1} \in P_{c}$ and $|t| \leq 2 \sqrt{\varepsilon}$ such that

$$
\Phi^{-t}\left(\theta_{1}, v\left(\theta_{1}\right)\right)=\theta_{0},
$$

where $\Phi^{t}(\theta, v)$ is the solution of the Euler-Lagrange equation of (6) with initial position $\theta$ and initial velocity $v$. To that end we rewrite (16) as

$$
\theta_{1}-t v\left(\theta_{1}\right)+R\left(t, \theta_{1}\right)=\theta_{0} ;
$$

$R$ is $C^{1}$-small. The last equality defines $\theta_{0}$ as the function of $\theta_{1} \in P_{c}$ and $t$ in a small neighborhood of zero. The linearization of this map at the point of tangency of $P_{c}$ with $\mathcal{L}_{0}$ is nonsingular since $v$ is transversal to $P_{c}$. By the implicit function theorem, the inverse map, i.e., the map from $\theta_{0} \in \mathcal{L}_{0}$ to the pair $\left(t, \theta_{1}\right), \theta_{1} \in P_{c}$, is well defined on a small neighborhood of the point of tangency, which for small $\varepsilon$ includes $\mathcal{L}_{0}$.

We now prove that the semi-trajectory $\gamma_{c}^{+}\left(\left[t_{\text {exit }}, \infty\right)\right)$, where $t_{\text {exit }}$ is the first exit time from $\mathcal{L}_{0}$, meets no other scatterers $\mathcal{L}$. To that end we observe that the $z$-coordinate $z(t)$ of $\gamma_{c}^{+}(t)$ satisfies the pendulum equation with energy fixed $H^{z}(z, \dot{z})=\frac{\dot{z}^{2}}{2}-\varepsilon \cos ^{2}\left(\frac{\pi z}{2}\right)=E$, where $E=0$ since $z \rightarrow 1$, $\dot{z} \rightarrow 0$ as $t \rightarrow \infty$. Thus

$$
\dot{z}=\sqrt{2 \varepsilon} \cos \left(\frac{\pi z}{2}\right)>1.2 \sqrt{\varepsilon} \text { if }|z|<\sqrt{\varepsilon} .
$$

Moreover, the horizontal speed $|(\dot{x}, \dot{y})|=\left|\left(c_{1}, c_{2}\right)\right|=1$, and hence the slope

$$
\frac{\dot{z}}{|(\dot{x}, \dot{y})|}>1.2 \sqrt{\varepsilon} \text { inside }|z|<\sqrt{\varepsilon} \text {. }
$$

This shows that any trajectory $\gamma_{c}^{+}$starting inside an $\sqrt{\varepsilon}$-ball does not intersect any other scatterers.

This completes the proof of parts (1) and (2) of Lemma 1. Part 3 of the lemma can be proven similarly. In the unperturbed case, existence and uniqueness of the minimizer follows from explicit integrability. In the perturbed case, the implicit function theorem applies.

Lemma 2. There exists $\varepsilon_{0}>0$ such that for all $0<\varepsilon<\varepsilon_{0}$ the following holds. If $n_{0}, n_{1} \in 2 \mathbb{Z}^{3}$ and $T \in \mathbb{R}$ satisfy $\pi_{3}\left(n_{1}-n_{0}\right)=2, T>\varepsilon^{-k-3}$ and

$$
\left|n_{1}-n_{0}-c T-(0,0,2)\right|<\sqrt{\varepsilon},
$$

then for any $\theta_{i} \in \mathcal{L}_{i}$ (where $\mathcal{L}_{i}$ is centered at $n_{i}, i=0,1$ ) and for all $|\tau-T|<1$ we have

$$
\left|A_{c}^{\tau}\left(\theta_{0}, \theta_{1}\right)-A_{c}^{+}\left(\theta_{0}\right)-A_{c}^{-}\left(\theta_{1}\right)\right| \leq \frac{1}{T} .
$$

Moreover, the function $A_{c}^{\tau}\left(\theta_{0}, \theta_{1}\right)$ is smooth in $\theta$ and $\tau$ and its minimum with respect to $\tau$ satisfies

$$
\min _{|\tau-T| \leq 25 \sqrt{\varepsilon}} A_{c}^{\tau}\left(\theta_{0}, \theta_{1}\right)<\min _{25 \sqrt{\varepsilon} \leq|\tau-T| \leq 1} A_{c}^{\tau}\left(\theta_{0}, \theta_{1}\right)-\frac{25 \varepsilon}{T} .
$$



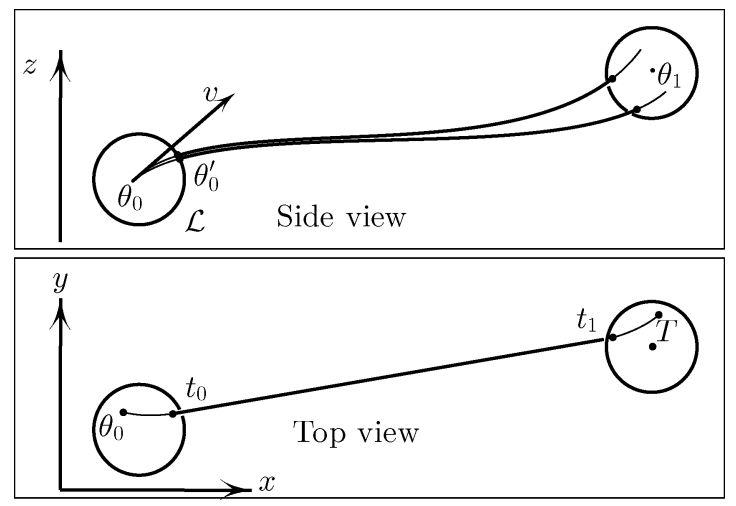

Figure 4. Dependence of trajectories on the endpoints is exponentially small in the $z$-direction and algebraically (like $O(1 / T)$ ) small in the $(x, y)$-direction.

Proof. Let $\tau$ satisfy $|\tau-T|<1$. We decompose the action $A_{c}^{\tau}$ into the $x y$ component, the rest

$$
\begin{aligned}
A_{c}^{\tau}\left(\theta_{0}, \theta_{1}\right)=\int_{0}^{\tau} L_{c}(\gamma(s), \dot{\gamma}(s), \varepsilon) d s & =\int_{0}^{\tau}\left(\frac{\left(\dot{x}(s)-c_{1}\right)^{2}}{2}+\frac{\left(\dot{y}(s)-c_{2}\right)^{2}}{2}\right) d s \\
+\int_{0}^{\tau}\left(\frac{\dot{z}^{2}(s)}{2}+\varepsilon U(\gamma(s), \varepsilon)\right) d s & =\int_{\gamma} \mathbf{1}+\int_{\gamma} \mathbf{2}
\end{aligned}
$$

with similar decompositions for $A_{c}^{+}\left(\theta_{0}\right)$ and $A_{c}^{-}\left(\theta_{1}\right)$, and show that the corresponding components match within the error claimed in (18). By Lemma 1 part (3) there exists a unique minimizer $\gamma(s)=(x(s), y(s), z(s)), 0 \leq s \leq \tau$, with $\gamma(0)=\theta_{0}$ and $\gamma(\tau)=\theta_{1}$. Lemma 1 implies that $\gamma$ exits $\mathcal{L}_{0}$ at some time $s=t_{0}>0$ and enters $\mathcal{L}_{1}$ at $s=t_{1}$ without meeting any other scatterers for $s \in\left(t_{0}, t_{1}\right)$ (see Figure 4).

We show first that the $(x, y)$-part of the action over $\left(t_{0}, t_{1}\right)$ is less than const $\frac{\varepsilon}{\tau}$.

Since $|(\dot{x}, \dot{y})| \geq \frac{4}{5}$ on $(0, \tau)$, and since the diameter of $\mathcal{L}_{i}$ is $2 \sqrt{\varepsilon}$, we have $t_{0}$, $\tau-t_{1} \leq 2.5 \sqrt{\varepsilon}$, and hence we have

$$
t_{1}-t_{0}=\tau+" 5 \sqrt{\varepsilon} ",
$$

where now and in the future " $X$ " stands for a term bounded by $X$ in absolute value. Since the minimizer does not meet any scatterers between $\mathcal{L}_{0}$ and $\mathcal{L}_{1}$, we have

$$
(\dot{x}, \dot{y})=\text { const for } t \in\left(t_{0}, t_{1}\right),
$$

and thus

$$
\left.(x, y)\right|_{t_{0}} ^{t_{1}}=(\dot{x}, \dot{y})(\tau+" 5 \sqrt{\varepsilon} ")=\bar{n}_{1}-\bar{n}_{0}+" 2 \sqrt{\varepsilon} ",
$$

where $\bar{n}_{i}$ denotes the $(x, y)$-component of $n_{i}$. Thus, we obtain a horizontal velocity estimate

$$
(\dot{x}, \dot{y})-c=\frac{\bar{n}_{1}-\bar{n}_{0}+" 3 \sqrt{\varepsilon} "}{\tau+" 5 \sqrt{\varepsilon} "}-c \stackrel{17}{=} \frac{T-\tau+" 9 \sqrt{\varepsilon} "}{\tau+" 5 \sqrt{\varepsilon} "} .
$$


Let $|\tau-T| \leq D \sqrt{\varepsilon}$ for some $D>4$. Substituting the last estimate into the $(x, y)$-part of the action over $\left(t_{0}, t_{1}\right)$, we get

$$
\frac{1}{2} \int_{t_{0}}^{t_{1}}((\dot{x}, \dot{y})-c)^{2} d t \leq \frac{(10+D)^{2}}{2} \frac{\varepsilon}{T^{2}} T=\frac{(10+D)^{2}}{2} \frac{\varepsilon}{T} .
$$

For the one-sided action $A_{c}^{+}\left(\theta_{0}\right)$, we have $(\dot{x}, \dot{y})=c$ outside $\mathcal{L}_{0}$, so that the corresponding integral vanishes. Letting $D=\varepsilon^{-1 / 2}$, we conclude that the difference of the first components outside $\mathcal{L}_{0} \cup \mathcal{L}_{1}$, or, equivalently, the first component $\int_{\gamma} \mathbf{1}$ outside $\mathcal{L}_{0} \cup \mathcal{L}_{1}$, is bounded by $\frac{2}{3 T}$ (see Figure 4 , bottom part).

Now, if $|\tau-T| \geq 25 \sqrt{\varepsilon}$, then (21) yields

$$
\frac{1}{2} \int_{t_{0}}^{t_{1}}((\dot{x}, \dot{y})-c)^{2} d t \geq 125 \frac{\varepsilon}{T^{2}} T=125 \frac{\varepsilon}{T} \text {. }
$$

We now show that the $(x, y)$-integrals inside $\mathcal{L}_{0} \cup \mathcal{L}_{1}$ satisfy the desired bound (18). Let $v=\dot{\gamma}\left(t_{0}\right)$ be the exit velocity from $\mathcal{L}_{0}$ for the minimizer of $A^{T}\left(\theta_{0}, \theta_{1}\right)$, and let $v^{+}$be the exit velocity from $\mathcal{L}_{0}$ of the minimizer of $A_{c}^{+}\left(\theta_{0}\right)$. We have

$$
\left|v-v^{+}\right| \leq \operatorname{const} \frac{\sqrt{\varepsilon}}{T}
$$

as was already proven for the horizontal components, and will be shown shortly for the $z$-components. By II. A), we have

$$
\left|\gamma-\gamma^{+}\right|_{C^{1}\left[0, t_{0}\right]} \leq \text { const } \frac{\sqrt{\varepsilon}}{T}
$$

and hence the difference between the $(x, y)$-integrals of $A_{c}^{T}$ and $A_{c}^{+}$inside $\mathcal{L}_{0}$ is bounded by $t_{0} \frac{\varepsilon}{T^{2}} \leq$ const $\frac{\varepsilon^{\frac{3}{2}}}{T^{2}}$.

To complete the proof of (18), we compare the $z$-components of action $\int_{\gamma} 2$ for $z$ and $z^{+}$. As before, let $z=z(t)$ be the $z$-component of the minimizer of $A_{c}^{T}\left(\theta_{0}, \theta_{1}\right)$. For $t \in\left(t_{0}, t_{1}\right)$ the energy (9) is conserved, and the phase flow in the $(z, \dot{z})$-plane is as shown in Figure 3. Since $z(t)$ is confined to a fixed neighborhood of the saddle for the duration $t_{1}-t_{0}=O(T)$, we conclude that

$$
0<H^{z}(z(t), \dot{z}(t))=O\left(\exp \left(-\frac{\sqrt{\varepsilon \pi} T}{2}\right)\right) \text { for } t_{0} \leq t \leq t_{1} .
$$

Note that $\left(z^{+}, \dot{z}^{+}\right)$, where $z^{+}=\pi_{3} \gamma^{+}$, travels along the stable manifold of the saddle, while in the strip $\frac{1}{4} \leq z \leq 1$.

The last two observations show that the orbits of $z(t)$ and $z^{+}(t)$ in the phase space are exponentially close, from which one concludes that the $z$-parts of the actions in (18) are exponentially small.

To prove the estimate (19), notice that the minimum with respect to $\tau$ for $|\tau-T|<4 \sqrt{\varepsilon}$ is bounded from below by the one for $|\tau-T|<25 \sqrt{\varepsilon}$. Thus it suffices to prove (19) with minimization in the left-hand side replaced by $|\tau-T|<4 \sqrt{\varepsilon}$. Pick $\tau$ and $\tau^{\prime}$ such that $|\tau-T|<4 \sqrt{\varepsilon}$ and $\left|\tau^{\prime}-T\right|>25 \sqrt{\varepsilon}$. We compare actions (20) for $\tau$ and $\tau^{\prime}$ componentwise. Using (22) with $D=4$ we have $\int_{\gamma} \mathbf{1}<98 \frac{\varepsilon}{T}$ for $\tau$. Using (23) we have $\int_{\gamma} \mathbf{1}>125 \frac{\varepsilon}{T}$ for $\tau^{\prime}$. This shows that the first components differ by at least $\frac{27 \sqrt{\varepsilon}}{T}$. Inside both scatterers action is bounded by const $\frac{\varepsilon^{\frac{3}{2}}}{T^{2}}$. The $z$-components of both actions for $\tau$ and $\tau^{\prime}$ are exponentially small due to (25). To 
summarize, all the terms of action except the first ones outside the scatterers are negligibly small. This proves (19) and completes the proof of the lemma.

Lemma 3. In the above notation for any $c=\left(c_{1}, c_{2}, 0\right),|c|=1$, and all sufficiently small $\varepsilon>0$ we have

$$
\min _{\theta \in S_{c},|\theta|<\sqrt{\varepsilon}} A_{c}(\theta)<\min _{\theta \in S_{c},|\theta|=\sqrt{\varepsilon}} A_{c}(\theta)-\frac{4 \varepsilon^{k+2}}{5},
$$

where $S_{c}$ is a 2-dimensional disk formed by intersection of the plane orthogonal to $c$ with the $\sqrt{\varepsilon}$-ball centered at the origin.

Proof. We pick $\theta \in S_{c}$ and $\theta^{\prime} \in \partial S_{c}$, and compare improper integrals representing $A_{c}$. We reduce the difference of these improper integrals to the difference of integrals over finite intervals. If $\theta \in \partial S_{c}$, then $\gamma_{c}^{ \pm}(\theta)$ does not enter the $\varepsilon$-ball around the origin. Therefore, this $c$-minimizer is also a trajectory of the integrable EulerLagrange flow of (15) as was shown in Lemma 1, part (2), and

$$
A_{c}(\theta)=\int_{-\infty}^{\infty}\left(\frac{\dot{z}^{2}(t)}{2}+\varepsilon \cos ^{2}\left(\frac{\pi z(t)}{2}\right)\right) d t,
$$

where $z(t) \rightarrow \pm 1$ as $t \rightarrow \pm \infty$ (see Figure [3).

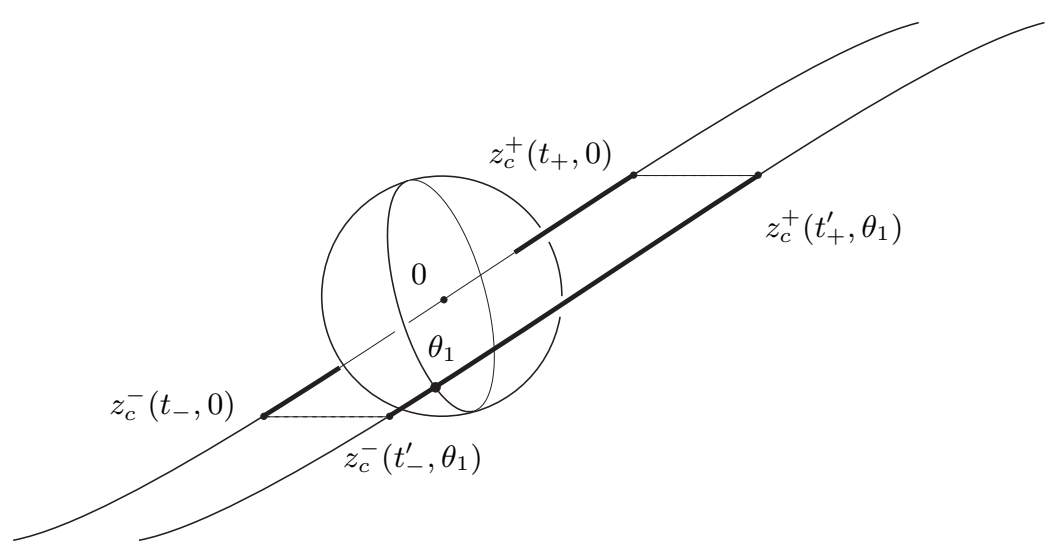

Figure 5

In view of the above arguments, it suffices to compare $A_{c}\left(\theta_{i}\right), i=0,1$, for $\theta_{0}=0$ and $\theta_{1} \in \partial S_{c}$. Let $t_{+}>0$ be the smallest $t$ for which the $z$-component of $\gamma_{c}^{+}(t, 0)$ reaches $\varepsilon$ : $z_{c}^{+}\left(t_{+}, 0\right)=\varepsilon$. Similarly, we define $t_{+}^{\prime}$ by the condition $z_{c}^{+}\left(t_{+}^{\prime}, \theta_{1}\right)=\varepsilon$; analogously, we define the times $t_{-}$and $t_{-}^{\prime}$ (see Figure 51). Outside the thickened segments the corresponding segments of $\gamma_{c}^{+}\left(t_{+}+t, 0\right)$ and $\gamma_{c}^{+}\left(t_{+}^{\prime}+t, \theta_{1}\right)$ for $t \geq 0$ coincide up to $(x, y)$-translation, and hence the actions along these trajectories are the same; a similar statement holds for the left semi-infinite pieces. It remains to compare the actions along the thickened segments.

To that end we note that velocity $\dot{\gamma}_{c}^{+}\left(t_{+}, 0\right)=\dot{\gamma}_{c}^{+}\left(t_{+}^{\prime}, \theta_{1}\right)$ and $\dot{\gamma}_{c}^{-}\left(t^{-}, 0\right)=$ $\dot{\gamma}_{c}^{-}\left(t_{-}^{\prime}, \theta_{1}\right)$. By II. B), we have

$$
\left|\dot{\gamma}_{c}^{+}\left(t_{+}-\tau, 0\right)-\dot{\gamma}_{c}^{+}\left(t_{+}^{\prime}-\tau, \theta_{1}\right)\right|=O\left(\tau \varepsilon^{k}\right) .
$$


This implies that for $\tau=O(\varepsilon)$, we have

$$
\mid \gamma_{c}^{+}\left(t_{0}-\tau, 0\right)-\gamma_{c}^{+}\left(t_{0}^{\prime}-\tau, \theta_{1}\right)+\text { const } \mid=O\left(\varepsilon^{k+2}\right),
$$

where the vector const has zero $z$-component. Heuristically, the two curves almost coincide (up to an $(x, y)$-translation), and thus their actions will differ due to the $\beta$-term, which contributes to the action along only one of these curves. Specifically, we split the Lagrangian $L_{c}$ into three parts:

$$
\begin{aligned}
L_{c}(x, y, z, \dot{x}, \dot{y}, \dot{z}) & =\frac{((\dot{x}, \dot{y})-c)^{2}}{2}+\left(\frac{\dot{z}^{2}}{2}+\varepsilon \cos ^{2}\left(\frac{\pi z}{2}\right)\right)+\left(-\varepsilon^{k+1} \beta(\theta, \varepsilon)\right) \\
& =: I+I I+I I I .
\end{aligned}
$$

Componentwise comparison proves the desired estimate (26).

\section{Shadowing, or "DifFusing", trajeCtories}

In this section we complete the proof of Theorem 1. The idea of construction of diffusing trajectories is to find a variational problem whose solution is a trajectory of the Euler-Lagrange flow with properties stated in this theorem. For the convenience of the reader we shall proceed in two steps. First we construct an auxiliary variational problem (29) and prove it has a solution satisfying the equations of motion (2) apart from finitely many "corners". We then modify the variational problem (see (37)) so that the corresponding solution is a true solution of the Euler-Lagrange equations and that it $\varepsilon$-shadows the pseudo-trajectory.

We introduce some notation used in the proof of Theorem 1 .

Fix small $\varepsilon>0$, and let $m=O\left(\varepsilon^{-k-3}\right), \rho=\varepsilon^{k+3}$ and $T_{0}=\varepsilon^{-k-3}$. Consider sequences of:

- asymptotic velocity directions $\left\{c_{j}=\left(c_{j}^{1}, c_{j}^{2}, 0\right),\left|c_{j}\right|=1\right\}_{j=0}^{m}$ such that $\left|c_{j+1}-c_{j}\right|<\rho$ for each $j=1, \ldots, m-2$ with $c_{0}=c_{1}$ and $c_{m-1}=c_{m}$;

- pairs of integers $\Delta n_{j}=\left(\Delta n_{j}^{1}, \Delta n_{j}^{2}\right)$ and times $T_{0}<T_{j}<2 T_{0}$ such that $\left|\left(\Delta n_{j}^{1}, \Delta n_{j}^{2}\right)-c T_{j}\right|<\sqrt{\varepsilon}$; let $n_{j+1}=n_{j}+\Delta n_{j}$ with $n_{0}=(0,0)$, and consider scatterers $\mathcal{L}_{0}, \ldots, \mathcal{L}_{m} \subset \mathbb{R}^{3}$ such that $\mathcal{L}_{j+1}=\mathcal{L}_{j}+\left(\Delta n_{j}, 2\right)$; we recall that $\mathcal{L}_{0}$ is the $\sqrt{\varepsilon}$-ball centered at the origin in $\mathbb{R}^{3}$.

Let $S_{j}=\left\{\theta: \theta \in \mathcal{L}_{n_{j}}, \theta(\bmod 2) \cdot c_{j}=0\right\}$ be the 2-dimensional disk of radius $\sqrt{\varepsilon}$ concentric with $\mathcal{L}_{n_{j}}$ for $j=0, \ldots, m$. Denote the set $\left\{\left(c_{j}, n_{j}, S_{j}, T_{j}\right), j=0 \ldots, m\right\}$ by $\mathcal{I}_{m}$.

Let $Q$ be the hypercube $Q=\left\{(\theta, \tau)=\left(\theta_{0}, \ldots, \theta_{m}, \tau_{0}, \ldots, \tau_{m-1}\right): \theta_{j} \in S_{j}\right.$, $\left.\left|\tau_{j}-T_{j}\right|<1\right\}$.

Introducing the discretized $c$-action

$$
\mathcal{A}(\theta, \tau, c)=A_{c_{0}}^{-}\left(\theta_{0}\right)+\sum_{j=0}^{m} A_{c_{j}}^{\tau_{j}}\left(\theta_{j}, \theta_{j+1}\right)+A_{c_{m}}^{+}\left(\theta_{m}\right),
$$

we minimize this action over $Q$ :

$$
\mathcal{M}_{\mathcal{I}_{m}}=\min _{Q} \mathcal{A}(\theta, \tau, c) .
$$

Lemma 4. Let the set $\mathcal{I}_{m}$ satisfy the conditions above. Then the minimum in (30) is attained in the interior of the hypercube $Q$. The value $\mathcal{M}_{\mathcal{I}_{m}}$ is positive and finite. Moreover, there is a shadowing trajectory $\gamma(t)$ of the Euler-Lagrange flow of (6) such that $\gamma\left(t_{j}\right)$ passes through the sections $S_{j}, j=0, \ldots, m$, and $\dot{\gamma}(t) \rightarrow c_{m}$ as $t \rightarrow+\infty$ and $\dot{\gamma}(t) \rightarrow c_{0}$ as $t \rightarrow-\infty$. 
Remark 2.1. This lemma implies the first part of Theorem 1, To prove the second part, one can choose $c_{0}=-c_{m}$ (which requires $m=O\left(\varepsilon^{k+3}\right)$ ), thus producing a direction reversing trajectory.

Proof. By Lemma 1, part (2), the barrier $A_{c}^{ \pm}(\theta)$ is continuous in $\theta$ and $c$ and by Lemma 2 action $A_{c_{j}}^{\tau_{j}}\left(\theta_{j}, \theta_{j+1}\right)$ is continuous in $\theta$ 's and $c_{j}$, so that $\mathcal{A}(\theta, \tau, c)$ in (30) is a continuous function of $(\theta, \tau)$. Since the hypercube $Q$ is compact, a minimum is attained. Our goal is to show that any minimum occurs in the interior and, moreover,

Note that

$$
\mathcal{M}_{\mathcal{I}_{m}}+\frac{\varepsilon^{k+2}}{2}<\min _{(\theta, \tau) \in \partial Q} \mathcal{A}(\theta, \tau, c) .
$$

$$
\mathcal{M}_{\mathcal{I}_{m}}=\min _{\theta: \theta_{j} \in S_{j}} \min _{\tau:\left|\tau_{j}-T_{j}\right| \leq 1} \mathcal{A}(\theta, \tau, c) .
$$

For any $j$ with $0<j<m$, the triple $\left(c_{j}, n_{j}, T_{j}\right)$ satisfies the conditions of Lemma 2] By (19) in Lemma 2 for any $\theta_{j-1} \in \mathcal{L}_{j-1}$ and $\theta_{j} \in \mathcal{L}_{j}$, the minimum with respect to $\tau_{j}$ for $\left|\tau_{j}-T_{j}\right|<1$ satisfies $\left|\tau_{j}-T_{j}\right| \leq 25 \sqrt{\varepsilon}$ and hence belongs to the interior of constraints.

Now consider minimization with respect to $\theta$. Fix $j: 0<j<m$ and $\tau_{j-1}, \tau_{j}$ satisfying $\left|\tau_{i}-T_{i}\right|<1$ for $i=j-1, j$ and $\theta_{j \pm 1} \in S_{j \pm 1}$. Denote the sum of the terms containing $\theta_{j}$ by

$$
s_{j}\left(\theta_{j}\right)=A_{c_{j-1}}^{\tau_{j-1}}\left(\theta_{j-1}, \theta_{j}\right)+A_{c_{j}}^{\tau_{j}}\left(\theta_{j}, \theta_{j+1}\right) .
$$

It suffices to show that the minimum of $s_{j}$ with respect to $\theta_{j} \in S_{j}$ occurs in the interior of $S_{j}$.

By (18) we have

$$
s_{j}\left(\theta_{j}\right)=A_{c_{j-1}}^{+}\left(\theta_{j-1}\right)+A_{c_{j-1}}^{-}\left(\theta_{j}\right)+A_{c_{j}}^{+}\left(\theta_{j}\right)+A_{c_{j}}^{-}\left(\theta_{j+1}\right)+{ }^{*} 2 \varepsilon^{k+3} ",
$$

recalling that " $X$ " denotes a term bounded by $X$ in absolute value. Since we have $\left|c_{j}-c_{j-1}\right|<\rho=\varepsilon^{k+3}$, by Lemma 1 part (2), the $c$-derivative of $A_{c}^{+}(\theta)$ is bounded uniformly in $\varepsilon$, and thus

$$
A_{c_{j-1}}^{+}\left(\theta_{j}\right)+A_{c_{j}}^{-}\left(\theta_{j}\right)=A_{c_{j}}\left(\theta_{j}\right)+\text { const " } \varepsilon^{k+3} \text { ". }
$$

By Lemma 3

$$
\min _{\theta_{j} \in \partial S_{j}} A_{c_{j}}\left(\theta_{j}\right)>\min _{\theta_{j} \in S_{j}} A_{c_{j}}\left(\theta_{j}\right)+\frac{4 \varepsilon^{k+2}}{5} .
$$

This shows that

$$
\min _{\theta_{j} \in S_{j}} s\left(\theta_{j}\right)<\min _{\theta_{j} \in \partial S_{j}} s\left(\theta_{j}\right)-\text { const } \varepsilon^{k+2}
$$

and the minimum of $s_{j}\left(\theta_{j}\right)$ is achieved in the interior of $\theta$-constraints for any fixed $\tau$. This implies the interior property of the minimizer of (29) and proves the first part of the lemma. We now prove the existence of the shadowing trajectory. The curve $\gamma(t)$, which corresponds to the minimizer of the $c$-action (29) satisfies the Euler-Lagrange equation on each time segment $\left(t_{j}^{*}, t_{j+1}^{*}\right)$, where $t_{j+1}=t_{j}+\tau_{j}$ for $j=0, \ldots, m-1$. However, there is no reason to expect that the derivatives match at $t=t_{j}^{*}$. We will show, however, that in the vicinity of this pseudo-solution there exists a true solution. We will do so by Mather's method of modifying the $c$-action 
(29) so that its critical point corresponds to a true trajectory, while keeping this modification small enough to preserve the existence of an interior minimizer.

For each $j=1, \ldots, m-1$, we define $\bar{\theta}_{j}=\theta-n_{j+1}$ and an exact one-form $\eta_{j}: T \mathbb{R}^{3} \rightarrow \mathbb{R}, \eta_{j}(\theta, v)=\left\langle\nabla b_{j}(\theta), v\right\rangle$ where $b_{j}: \mathbb{R}^{3} \rightarrow \mathbb{R}$ is given by

$$
b_{j}(\theta)= \begin{cases}c_{j} \cdot \theta & \text { for }\left|\bar{\theta}_{j}\right| \geq \varepsilon, \\ c_{j+1} \cdot \theta & \text { for }\left|\bar{\theta}_{j}\right| \leq 0.5 \varepsilon, \\ \left(\mu\left(\left|\bar{\theta}_{j}\right|\right) \cdot c_{j}+\left(1-\mu\left(\left|\bar{\theta}_{j}\right|\right)\right) c_{j+1}\right) \cdot \theta & \text { for } 0.5 \varepsilon \leq\left|\bar{\theta}_{j}\right| \leq 0.8 \varepsilon,\end{cases}
$$

where $\mu$ is a smooth nondecreasing function whose support is $(0.5, \infty)$ and which is identically one on $(0.8, \infty)$. Define

$$
L_{\eta_{j}}(\theta, \dot{\theta}, \varepsilon)=L(\theta, \dot{\theta}, \varepsilon)-\left\langle\nabla b_{j}(\theta), \dot{\theta}\right\rangle+\frac{\left\langle c_{j}, c_{j}\right\rangle}{2} .
$$

Since the one-form $\eta_{j}$ is closed, the Euler-Lagrange flows $L_{c_{j}}$ and $L_{\eta}$ coincide.

We replace the integrands $L_{c_{j}}$ by $L_{\eta_{j}}$ in (29), obtaining the modified $\eta$-action

$$
\mathcal{A}_{\eta}(\theta, \tau)=A_{\eta_{0}}^{-}\left(\theta_{0}\right)+\sum_{j=0}^{m} A_{\eta_{j}}^{\tau_{j}}\left(\theta_{j}, \theta_{j+1}\right)+A_{\eta_{m}}^{+}\left(\theta_{m}\right)
$$

We show first that an interior minimum of this action (over the same set of $\{(\theta, \tau)\}$ as in (31) $)$ corresponds to a solution of the Euler-Lagrange equation over the whole $\mathbb{R}$. To that end it suffices to show that

$$
\frac{\partial}{\partial \theta_{j}}\left(A_{\eta_{j-1}}^{\tau_{j-1}}\left(\theta_{j-1}, \theta_{j}\right)+A_{\eta_{j}}^{\tau_{j}}\left(\theta_{j}, \theta_{j+1}\right)\right)=0
$$

implies

$$
\frac{\partial}{\partial \theta_{j}}\left(A_{0}^{\tau_{j-1}}\left(\theta_{j-1}, \theta_{j}\right)+A_{0}^{\tau_{j}}\left(\theta_{j}, \theta_{j+1}\right)\right)=0 .
$$

The last relation expresses the continuity of momentum, $\dot{\gamma}\left(t_{j}-\right)=\dot{\gamma}\left(t_{j}+\right)$; indeed, $\dot{\gamma}\left(t_{j}-\right)=\frac{\partial}{\partial \theta} \mathcal{A}^{\tau}\left(\theta_{j-1}, \theta\right)_{\theta=\theta_{j}}$ and $\dot{\gamma}\left(t_{j}+\right)=-\frac{\partial}{\partial \theta} \mathcal{A}^{\tau}\left(\theta, \theta_{j+1}\right)_{\theta=\theta_{j}}$. To prove the implication (38) $\Rightarrow$ (39), we observe that by the definition of $A_{\eta}$ we have

$$
\begin{gathered}
A_{\eta_{j-1}}^{\tau_{j-1}}\left(\theta_{j-1}, \theta_{j}\right)+A_{\eta_{j}}^{\tau_{j}}\left(\theta_{j}, \theta_{j+1}\right) \\
=A_{0}^{\tau_{j-1}}\left(\theta_{j-1}, \theta_{j}\right)+A_{0}^{\tau_{j}}\left(\theta_{j}, \theta_{j+1}\right)+\left(b_{j-1}\left(\theta_{j}\right)-b_{j-1}\left(\theta_{j-1}\right)\right)+\left(b_{j}\left(\theta_{j+1}\right)-b_{j}\left(\theta_{j}\right)\right) .
\end{gathered}
$$

By the construction of $\eta_{j}$ we have $\eta_{j}\left(\theta_{j}\right)=\eta_{j+1}\left(\theta_{j}\right)$ for $\eta_{j}$ in the $\sqrt{\varepsilon}$-ball concentric with $S_{j}$, and thus the $\eta$-term in (39) does not depend on $\theta_{j}$, proving that (38) and (39) are equivalent for $\theta$ as indicated earlier.

Similar to $s_{j}\left(\theta_{j}\right)$ defined above, consider

$$
s_{j}^{\eta}\left(\theta_{j}\right)=A_{\eta_{j-1}}^{\tau_{j-1}}\left(\theta_{j-1}, \theta_{j}\right)+A_{\eta_{j}}^{\tau_{j}}\left(\theta_{j}, \theta_{j+1}\right) .
$$

We will show that

$$
\left|s\left(\theta_{j}\right)-s^{\eta}\left(\theta_{j}\right)\right|<3 \varepsilon^{k+4} .
$$

This would imply, in view of (35), that the minimum of (37) occurs in the interior.

Suppose $\gamma_{j}:\left[t_{j-1}, t_{j+1}\right] \rightarrow \mathbb{R}^{3}$ (resp. $\gamma_{j}^{\eta}:\left[t_{j-1}, t_{j+1}\right] \rightarrow \mathbb{R}^{3}$ ) is a minimizer of $s_{j}\left(\theta_{j}\right)$ (resp. $s_{j}^{\eta}\left(\theta_{j}\right)$ ) subject to all parameters fixed. In particular, we take $\gamma_{j}\left(t_{j}\right)=\gamma_{j}^{\eta}\left(t_{j}\right)=\theta_{j}$. If $\theta_{j} \in \partial S_{j}$, then by Lemma 1, part (3), $\gamma_{j}$ misses the

\footnotetext{
${ }^{9}$ Restriction of such a one-form to $T \mathbb{T}^{3}$ is a closed one-form.
} 


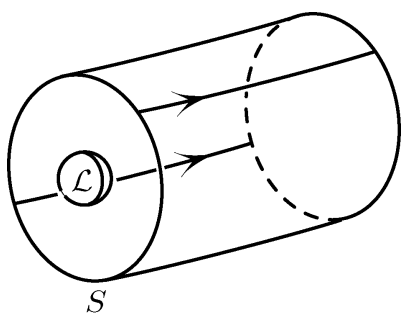

FiguRE 6. Trajectories starting on $\partial S$ avoid the scatterer $\mathcal{L}$ concentric with $S$.

scatterer at $n_{j}$; see Figure 6. Since $L_{\eta_{j}}$ and $L_{c_{j}}$ coincide outside the scatterer, we have $s_{j}\left(\theta_{j}\right)=s_{j}^{\eta}\left(\theta_{j}\right)$ for $\theta_{j} \in \partial S_{j}$.

Below we shall prove that a broken trajectory $\gamma=\gamma^{-} \cup \gamma^{+}$consisting of two parts, each satisfying the Euler-Lagrange equation on the respective intervals $\left[t_{j-1}, t_{j}\right]$ and $\left[t_{j}, t_{j+1}\right]$ with $\gamma^{-}\left(t_{j}\right)=\gamma^{+}\left(t_{j}\right)=\theta_{j} \in S_{j}$, satisfies

$$
\left|\int_{\gamma} L_{c_{j}}-\int_{\gamma} L_{\eta_{j}}\right|<3 \varepsilon^{k+4} .
$$

In particular, this estimate holds for $\gamma_{j}^{\eta}$ and $\gamma_{j}$. One can show that in fact $\gamma_{j}^{\eta}=\gamma_{j}$; however, we can get away without proving this, as follows.

We observe that (41) implies that

$$
\left|s\left(\theta_{j}\right)-\int_{\gamma_{j}} L_{\eta_{j}}\right|<3 \varepsilon^{k+4}
$$

and

$$
\left|s^{\eta}\left(\theta_{j}\right)-\int_{\gamma_{j}^{\eta}} L_{c_{j}}\right|<3 \varepsilon^{k+4} .
$$

By minimality we have $s\left(\theta_{j}\right) \leq \int_{\gamma_{j}^{\eta}} L_{c_{j}}$ and $s^{\eta}\left(\theta_{j}\right) \leq \int_{\gamma_{j}^{\eta}} L_{c_{j}}$. The last four inequalities imply (40). Since in the right-hand side $s\left(\theta_{j}\right)=s^{\eta}\left(\theta_{j}\right)$, we have

$$
\min _{\theta_{j} \in S_{j}} s^{\eta}\left(\theta_{j}\right)<\min _{\theta_{j} \in \partial S_{j}} s^{\eta}\left(\theta_{j}\right)-\text { const } \varepsilon^{k+2},
$$

this estimate is analogous to (35), and proves the interior property.

It remains to prove (41). Denote by $t_{j}^{-}$the entrance time to the $\varepsilon$-ball around $n_{j}$, i.e., maximal $t<t_{j}$ such that $\left|\gamma^{-}\left(t_{j}^{-}\right)-n_{j}\right|=\varepsilon$. Denote by $t_{j}^{+}$the exit from the $\varepsilon$ ball around $n_{j}$, i.e., the minimal $t_{j}^{+}>t$ such that $\left|\gamma^{+}\left(t_{j}^{+}\right)-n_{j}\right|=\varepsilon$. Outside of this $\varepsilon$-ball $L_{\eta_{j}}$ and $L_{c_{j}}$ coincide so actions are the same. To compare the actions inside the $\varepsilon$-ball, we recall the definition (36) and the estimate $\left|c_{j+1}-c_{j}\right| \leq \rho=\varepsilon^{k+3}$. By the Newton-Leibnitz formula,

$$
\int_{t_{j}^{-}}^{t_{j}^{+}} L_{\eta_{j}}(\gamma(t), \dot{\gamma}(t)) d t=\int_{t_{j}^{-}}^{t_{j}^{+}} L(\gamma(t), \dot{\gamma}(t)) d t+\left(b\left(t_{j}^{+}\right)-b\left(t_{j}^{-}\right)\right)+\frac{\left\langle c_{j}, c_{j}\right\rangle}{2}\left(t_{j}^{+}-t_{j}^{-}\right) .
$$

Comparing this integral with the one of $L_{c_{j}}$, we see that they differ only by $\left(b\left(t_{j}^{+}\right)-b\left(t_{j}^{-}\right)\right)-\left\langle c_{j}, t_{j}^{+}-t_{j}^{-}\right\rangle$. By the properties of $\nabla b$ this difference is bounded by $\varepsilon^{k+3}\left(t_{j}^{+}-t_{j}^{-}\right) \leq 3 \varepsilon^{k+4}$. This completes the proof of Lemma 4 . 


\section{MATHER's METHOD OF CHANGING THE LAGRANGIAN}

In this section we outline the general scheme of the proof of diffusion due to Mather [25].

Mather's proof of diffusion is variational rather than dynamic, and minimizers play the role of hyperbolic sets. In each cohomology class $c$, one defines the Mather, Aubry, and Mane sets $\dot{\mathcal{M}}_{c} \subset \dot{\mathcal{A}}_{c} \subset \dot{\mathcal{N}}_{c}$ in the phase space; see 20 and Remark 1.2. Rather than repeating various definitions from [4, 12, 14, 23, 28, we describe these sets in the phase space of our Lagrangian (6): $\dot{\mathcal{M}}_{c}=\dot{\mathcal{A}}_{c}$ are 2-tori in $\mathbb{T}^{3} \times \mathbb{R}^{3}$, given by $z=0, \dot{\theta}=c$. Projections $\mathcal{M}_{c}=\mathcal{A}_{c}$ of these tori onto the configuration space $\mathbb{T}^{3}$ are the 2-tori whose covering planes are $z=2 k$, for integer $k 10$ The Mane's set $\dot{\mathcal{N}}_{c}$ consists of orbits heteroclinic to $\dot{\mathcal{A}}_{c}$. In our example without the scatterers, the projection $\mathcal{N}_{c}$ onto $\mathbb{T}^{3}$ consists of the two-parameter family 11 of heteroclinic trajectories $\gamma_{c}$ (in addition to straight lines with direction $c$ in the planes $z=1(\bmod 2))$. Of the minimizers passing through scatterers, only isolated ones survive. In our very special example an open set of minimizers, namely the ones that do not pass through the scatterers, still survive; this is not a typical situation.

In our example, just as in the one of Arnold, the diffusing orbits follow the invariant manifolds of "whiskered tori" $z=1(\bmod 2), \dot{x}=c_{1}, \dot{y}=c_{2}, \dot{z}=0$. In Mather's proof the role of whiskered tori is picked up by Aubry sets $\dot{\mathcal{A}}_{c}$. The outline of Mather's proof is reflected in the treatment of our example. The main steps are the following.

(1) Transition quasiorbits. One proves that $W^{u}\left(\dot{\mathcal{A}}_{c}\right) \cap W^{s}\left(\dot{\mathcal{A}}_{c^{\prime}}\right) \neq \emptyset$ if $c, c^{\prime}$ are sufficiently close after a small perturbation of the flow, if necessary. This is done by picking an appropriate $(n-1)$-dimensional disk $S_{c} \in \mathbb{T}^{n} \backslash \mathcal{A}_{c}$ and defining the one-sided barrier function $A_{c}$ on this disk. One proves that any minimum of $A_{c}$ lies in the interior of the disk $S_{c}$.

(2) Regularity of the barrier function: In Lemma 1 part (2) we get regularity of the barrier function $A_{c}(\theta)$ in $\theta$ and $c$ nearly for free. In general, proving some, even weak, regularity in $c$ is a delicate problem (see [22], the twist map case), and sometimes it is even false (see [24]). However, recent work of Bernard-Contreras [5] gives us hope that for a generic Lagrangian it is true. We do use this regularity in the above proof (see (33)).

(3) Quasitrajectory. One forms a concatenated action as in (29). One fixes all $\theta_{i}$ but one $\left(\theta_{j}\right)$, and shows that the minimum of the concatenated action is achieved inside $S_{j}$ (regularity of the barrier function is used in this step). Then this implies the existence of a broken trajectory passing through the interior of every section.

(4) Shadowing trajectory. Finally, using the smallness of $c_{j-1}$ and $c_{j}$, one proves the existence a true trajectory in the vicinity of the quasitrajectory.

\footnotetext{
${ }^{10}$ In the old example of Hedlund [18 for most $c$ 's, the set $\mathcal{M}_{c}$ consists of only three geodesics directed along coordinate directions. This is in complete contrast with our example where the Aubry and Mather sets depend nicely on $c$.

${ }^{11}$ In the integrable case $(\beta=0)$ any $(x, y)$-translate of a heteroclinic $c$-minimizer is again a heteroclinic $c$-minimizer, hence the two parameters.
} 


\section{ABOUT THE AUTHORS}

Vadim Kaloshin is currently the Michael Brin Chair in Mathematics at University of Maryland at College Park. He was a recipient of an AIM five-year fellowship and an invited speaker at the 2006 ICM in Madrid.

Mark Levi is currently a professor at Pennsylvania State University.

\section{REFERENCES}

[1] Arnold, V. Mathematical methods of classical mechanics, Second edition, Graduate Texts in Mathematics, 60, 2nd edition, 1989. MR997295 (90c:58046)

[2] Arnold, V. Instabilities in dynamical systems with several degrees of freedom, Sov. Math. Dokl. 5 (1964), 581-585.

[3] Arnold, V. I.; Kozlov, V. V.; Neishtadt, A. I. Mathematical aspects of classical and celestial mechanics. Translated from the 1985 Russian original by A. Iacob. Reprint of the original English edition from the series Encyclopaedia of Mathematical Sciences [Dynamical systems. III], Encyclopaedia Math. Sci., 3, Springer, Berlin, 1993. Springer-Verlag, Berlin, 1997. MR 1656199 (2000b:37054)

[4] Bernard, P. Dynamics of pseudographs in convex Hamiltonian systems, to appear in the Journal of the AMS.

[5] Bernard, P.; Contreras, G. A generic property of families of Lagrangian systems, to appear in the Annals of Mathematics.

[6] Bessi, U. An approach to Arnold's diffusion through the calculus of vartiations, Nonlinear Anal. 26(6) (1996), 1115-1135. MR1375654 (97b:58123)

[7] Bessi, U.; Chierchia, L.; Valdinoci, E. Upper bounds on Arnold diffusion times via Mather theory, J. Math. Pures Appl. 80(1) (2001) 105-129. MR1810511(2002a:37093)

[8] Berti, M.; Bolle, Ph. A functional analysis approach to Arnold diffusion, Ann. Inst. H. Poincare 19(4) (2002), 395-450. MR.1912262 (2003g:37105)

[9] Bourgain J.; Kaloshin V. On diffusion in high-dimensional Hamiltonian systems. J. Funct. Anal. 229(1) (2005), 1-61. MR2180073

[10] Cheng, C.-Q.; Yan, J. Existence of diffusion orbits in a priori unstable Hamiltonian systems. J. Differential Geom. 67(3) (2004), 457-518. MR2153027 (2006d:37110)

[11] Cheng, C.-Q.; Yan J. Arnold diffusion in Hamiltonian systems: the a priori unstable case, preprint.

[12] Contreras, G.; Iturriaga, R. Global Minimizers of Autonomous Lagrangians. 22 Coloquio Brasileiro de Matematica, IMPA, Rio de Janeiro, 1999. MR1720372 (2001j:37113)

[13] Delshams, A.; de la Llave, R.; Seara, T. A geometric mechanism for diffusion in Hamiltonian systems overcoming the large gap problem: heuristics and rigorous verification on a model. Mem. Amer. Math. Soc. 179 (2006), no. 844, viii+141 pp. MR2184276

[14] Fathi, A. The weak KAM theorem in Lagrangian dynamics, Cambridge Studies in Advanced Mathematics, vol. 88, Cambridge Univesity Press, 2003.

[15] Fenichel, N. Persistence and smoothness of invariant manifolds for flows, Indiana Univ. Math. J. 21 (1971), 193-226. MR0287106 (44:4313)

[16] Hirsch, M.; Pugh, C.; Shub, M. Invariant manifolds, Lect. Notes in Math, vol. 583, Springer, 1977. MR0501173 (58:18595)

[17] Kaloshin, V.; Levi, M. Geometry of Arnold diffusion, to appear in SIAM Review.

[18] Levi, M. Shadowing property of geodesics in Hedlund's metric, Ergo. Th. \& Dynam. Syst. 17 (1997), 187-203.

[19] Marco, J.-P.; Sauzin, D. Stability and instability for Gevrey quasi-convex near-integrable Hamiltonian systems. Publ. Math. Inst. Hautes Etudes Sci. No. 96 (2002), 199-275 (2003). MR:1986314 (2004m:37112)

[20] Mather, J. Action minimizing invariant measures for positive definite Lagrangians, Math. Z. 207 (1991), 169-207. MR1109661 (92m:58048)

[21] Mather, J. Variational construction of connecting orbits, Ann. Inst. Fourier 43 (1993), 13491386. MR.1275203 (95c:58075)

[22] Mather, J. Modulus of continuity of Peierls' barrier, in NATO ASI Series C: vol. 209, Periodic Solutions of Hamiltonian Systems and Related Topics, edited by P. Rabinowitz, 1987, 177202. MR.920622 (89c:58109) 
[23] Mather, J. Arnold diffusion. I: Announcement of results, J. of Math. Sciences 124(5) (2004), 5275-5289. MR2129140 (2005m:37142)

[24] Mather, J. Total disconnectedness of the quotient Aubry set in low dimensions, Comm. Pure Appl. Math. 56(8) (2003), 1178-1183. MR1989233 (2004c:37152)

[25] Mather, J. Graduate Class 2001-2002, Princeton, 2002.

[26] Mather, J. Arnold diffusion. II, preprint, 2006, 160pp.

[27] Nekhoroshev, N. An exponential estimate of the time of stability of nearly integrable Hamiltonian systems, Uspekhi Math. Nauk 32(1) (1977), 5-66. MR0501140 (58:18570)

[28] Siburg, K. The Principle of Least Action in Geometry and Dynamics, Lecture Notes in Mathematics, 1844, Springer 2004. MR2076302 (2005m:37151)

[29] Treschev, D. Multidimensional symplectic separatrix maps, J. Nonlinear Sci. 12(1) (2002), 27-58. MR:1888569 (2002m:37089)

[30] Treschev, D. Evolution of slow variables in a priori unstable Hamiltonian systems. Nonlinearity 17(5) (2004), 1803-1841. MR2086152(2005g:37116)

[31] Xia, J. Arnold diffusion: a variational construction. Proc of the ICM, vol. II (Berlin, 1998), 867-877, 1998. MR1648133 (99g:58112)

Mathematics 253-37, California Institute of Technology, Pasadena, California 91125

E-mail address: kaloshin@math.psu.edu

Mathematics 253-37, California Institute of Technology, Pasadena, California 91125 